\title{
Modified Eccentric Connectivity Index and Polynomial of Tetragonal Carbon Nanocones $\mathrm{CNC}_{4}[n]$
}

\author{
Linli $\mathrm{Zhu}^{1,2}$, Wei $\mathrm{Gao}^{3}$ \\ ${ }^{1}$ School of Computer Engineering, Jiangsu University of Technology, Changzhou 213001, China \\ ${ }^{2}$ Jiangsu Key Laboratory of Recycling and Reuse Technology for Mechanical and Electronic Products, \\ Changshu 215500, China \\ ${ }^{3}$ School of Information Science and Technology, Yunnan Normal University, Kunming 650500, China \\ Email: zhulinli@jsut.edu.cn
}

\begin{abstract}
Chemical compounds and drugs are often modeled as graphs where each vertex represents an atom of molecule and covalent bounds between atoms are represented by edges between the corresponding vertices. This graph derived from a chemical compounds is called its molecular graph. The modified eccentric connectivity index defined over this molecular graph has been shown to be strongly correlated to oxidizing properties of the compounds. In this article, by virtue of molecular structural analysis, the modified eccentric connectivity index and modified eccentric connectivity polynomial of tetragonal carbon nanocones $\mathrm{CNC}_{4}[n]$ are reported. The theoretical results achieved in this article illustrate the promising prospects of the application to the chemical and pharmacy engineering.
\end{abstract}

Keywords: Theoretical chemistry, modified eccentric connectivity index, tetragonal carbon nanocone.

\section{Introduction}

Investigations of degree or distance based topological indices have been conducted over 35 years. Topological indices are numerical parameters of molecular graph, and play significant roles in physics, chemistry and pharmacology science. For example, the modified eccentric connectivity index reflects the oxidizing property of chemical compounds.

Specifically, let $G$ be a molecular graph, then a topological index can be regarded as a score function $f: G \rightarrow \mathbb{R}^{+}$, with this property that $f\left(G_{1}\right)=f\left(G_{2}\right)$ if $G_{1}$ and $G_{2}$ are isomorphic. As numerical descriptors of the molecular structure obtained from the corresponding molecular graph, topological indices have found several applications in theoretical chemistry, especially in QSPR/QSAR study. For instance, Wiener index, Zagreb index, harmonic index and sum connectivity index are introduced to reflect certain structural features of organic molecules. Several papers contributed to determine these distance-based indices of special molecular graph (See [1-10] for more details). The notation and terminology used but undefined in this paper can be found in $[11]$.

The graphs considered in this paper are simple and connected. The vertex and edge sets of $G$ are denoted by $V(G)$ and $E(G)$, respectively. The modified eccentric connectivity index of any graph was defined by Ashrafi and Ghorbani [12] as

$$
\zeta_{c}(G)=\sum_{v \in V(G)} M(v) e c(v)
$$

where $M(v)=\sum_{u \in N_{G}(v)} d(u)$ and $d(u)$ is the degree of vertex $u$ (the number of edges adjacent to $u$ ). The corresponding modified eccentric connectivity polynomial

$$
\zeta_{c}(G, x)=\sum_{v \in V(G)} M(v) x^{e c(v)} .
$$

Alaeiyan et al., [13] presented a numerical method for computing modified eccentric connectivity polynomial and modified eccentric connectivity index of one-pentagonal carbon nanocones. Ashrafi et al., [14] manifested several exact formulas for the modified eccentric connectivity polynomial of Cartesian product, symmetric difference, disjunction, and join of 
graphs.

In this paper, we obtain the modified eccentric connectivity polynomial of tetragonal carbon nanocones $\mathrm{CNC}_{4}[n]$.

\section{Eccentric Index of Tetragonal Carbon Nanocones $\mathrm{CNC}_{4}[n]$}

For the structure of tetragonal carbon nanocones $C N C_{4}[n]$ we can refer to Trinajstic [15] and Kumar and Modan [16]. By the graph structure analysis, we see that $\left|V\left(C N C_{4}[n]\right)\right|=4(n+1)^{2}$, $\left|E\left(C N C_{4}[n]\right)\right|=6 n^{2}+10 n+4, \quad \min \left\{e c\left(C N C_{4}[n]\right)\right\}=2 n+2, \quad$ and $\max \left\{e c\left(C N C_{4}[n]\right)\right\}=4 n+2$. Thus, all the eccentricities of vertices of $C N C_{4}[n]$ are varied between $2 n+2$ and $4 n+2$. Moreover, there are three classes of vertices in $C N C_{4}[n]: 4 n^{2}$ internal vertices of degree three with eccentricities between $2 n+2$ and $4 n$, and $4 n$ external vertices of degree 3 with eccentricities between $3 n+2$ and $4 n+2$, and $4 n+4$ external vertices of degree two with eccentricities between $3 n+2$ and $4 n+2$.

We determine the eccentric connectivity of $\mathrm{CNC}_{4}[n]$ in terms of algebraic trick, and consider two cases according to the parity of $n$. If $n \equiv 1(\bmod 2)$, see Figure 1 as an example, the external vertices of $\mathrm{CNC}_{4}[n]$ are made of $(n+1) / 2$ classes of vertices of degree 3 with eccentric connectivity equal to $3 n+2 k+2$ and $(n+1) / 2$ classes of vertices of degree 2 with eccentric connectivity equal to $3 n+2 k+3$, where $0 \leq k \leq(n-1) / 2$.

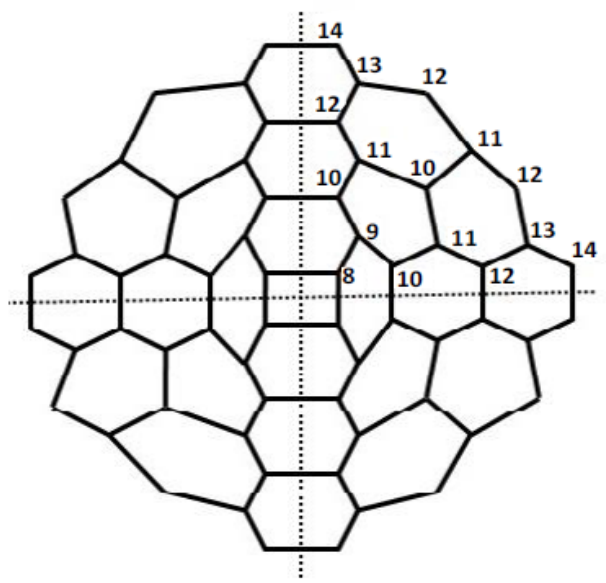

Figure 1. The eccentric connectivity of $\mathrm{CNC}_{4}[3]$.

If $n \equiv 0(\bmod 2)$, see Figure 2 as an example, the external vertices of $C N C_{4}[n]$ are made of $n / 2$ classes of vertices of degree 3 with eccentric connectivity equal to $3 n+2 k+3$ for $0 \leq k \leq(n-$ $2) / 2$ and $(n+2) / 2$ classes of vertices of degree 2 with eccentric connectivity equal to $3 n+2 k+2$, where $0 \leq k \leq n / 2$.

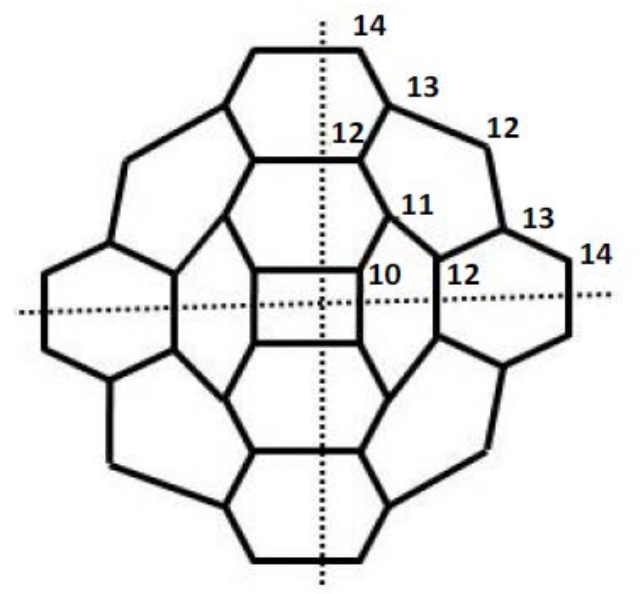

Figure 2. The eccentric connectivity of $\mathrm{CNC}_{4}[2]$. 
Moreover, by the structure analysis, there are four classes of vertex neighborhoods in $\mathrm{CNC}_{4}[n]$. The product of vertex neighbors degrees is equal to 27 for any internal vertex. Next, there are $4 n$ external vertices with degree 3 satisfying that the product of their neighbors degrees is equal to 12 . Then, there are exactly eight external vertices with degree 2 satisfying that their neighbors are of degrees 2 and 3 and for all of them, the sum of their neighbors degrees is equal to 6 . At last, there are $4 n-4$ vertices with degree 2 such that both neighbors of them are of degree 3 .

Now, the main result in this section is stated as follows:

Theorem 1. Let $\mathrm{CNC}_{4}[n]$ be the tetragonal carbon nanocones.

(1)If $n \equiv 1(\bmod 2)$, then

$$
\begin{aligned}
& \zeta_{c}\left(C N C_{4}[n]\right)=\frac{1}{48}\left(-21+6892 n+15450 n^{2}+2660 n^{3}+171 n^{4}\right), \\
& \zeta_{c}\left(C N C_{4}[n], x\right)=40 x^{4 n+2}+\sum_{l=0}^{\frac{n-3}{2}} \sum_{k=l}^{l+1}(54 k+8 l+56) x^{4 n-k-l+1}+ \\
& (36 n-8) x^{3 n+2}+36 n x^{3 n+1}+\sum_{l=0}^{\frac{n-3}{2}} \sum_{k=0}^{1} 9(3 n-k-2 l) x^{4 n-8 l-8} .
\end{aligned}
$$

(2) If $n \equiv 0(\bmod 2)$, then $\zeta_{c}\left(C N C_{4}[n]\right)=108 n^{3}+236 n^{2}+150 n+32$,

$$
\begin{aligned}
& \zeta_{c}\left(C N C_{4}[n], x\right)=\sum_{v \in V\left(C N C_{4}[n]\right)} M(v) x^{e c(v)}=\sum_{k=0}^{1} 8(2 k+5) x^{4 n+2-k}+ \\
& \sum_{l=0}^{\frac{n-4}{2}} \sum_{k=l}^{l+1}(64 l+120+8 k) x^{4 n-k-l}+(36 n+24) x^{3 n+2}+\sum_{l=0}^{\frac{n-2}{2}} \sum_{k=0}^{1} 9(3 n-k-2 l+1) x^{4 n-8 l-4}
\end{aligned}
$$

Proof. If $n \equiv 1(\bmod 2)$, see Figure 1 as an example, $C N C_{4}[n]=\bigcup_{i=1}^{n} T_{i}$, where $T_{i}$ is a partition of the molecular graph $C N C_{4}[n]$. There are four classes of vertices for each section of $T_{i}$. We infer eight vertices of class 1 with maximum eccentric connectivity $4 n+2$ and $M(u)=5$. Moreover, there are $8(n-2 l-2)$ vertices of class 2 for $0 \leq l \leq(n-3) / 2$. The eccentric connectivity of $4(n-2 l-2)$ vertices of them equals to $3 n-2 l$ and the eccentric connectivity of other vertices equals to $3 n-2 l-1$. For $l \leq k \leq l+1$, if $k=l$ then there are eight vertices satisfying $M(u)=7$ and $8 k$ vertices having $M(u)=9$. If $k-l=1$, then there are eight vertices such that $M(u)=6$ and $8 k$ vertices satisfying $M(u)=9$, and the eccentric connectivity of them is equals to $4 n-k-l+1$ where $0 \leq l \leq(n-3) / 2$. Furthermore, there are four vertices meeting $M(u)=7,4 n-4$ vertices satisfying $M(u)=9$ and $e c(u)=3 n+2$, and $4 n$ vertices having $M(u)=9$ and $e c(u)=3 n+1$. Hence, by the definition of modified eccentric connectivity index and modified eccentric connectivity polynomial, we have

$$
\begin{aligned}
& \zeta_{c}\left(C N C_{4}[n]\right)=\sum_{v \in V\left(C N C_{4}[n]\right)} M(v) e c(v)=40 \cdot(4 n+2)+\sum_{l=0}^{\frac{n-3}{2}} \sum_{k=l}^{l+1}(8 k \cdot 9+8(7-k+l)) \cdot(4 n-k-l+1) \\
& +(4 \cdot 7+(4 n-4) \cdot 9) \cdot(3 n+2)+36 n \cdot(3 n+1)+\sum_{l=0}^{\frac{n-3}{2}} \sum_{k=0}^{1} 9(3 n-k-2 l) \cdot(4 n-8 l-8) \\
& +\frac{1}{48}\left(-21+6892 n+15450 n^{2}+2660 n^{3}+171 n^{4}\right) \\
& \zeta_{c}\left(C N C_{4}[n], x\right)=\sum_{v \in V\left(C N C_{4}[n]\right)} M(v) x^{e c(v)}=40 x^{4 n+2}+\sum_{l=0}^{\frac{n-3}{2}} \sum_{k=l}^{l+1}(54 k+8 l+56) x^{4 n-k-l+1}+(36 n-8) x^{3 n+2} \\
& +36 n x^{3 n+1}+\sum_{l=0}^{\frac{n-3}{2}} \sum_{k=0}^{1} 9(3 n-k-2 l) x^{4 n-8 l-8}
\end{aligned}
$$

If $n \equiv 0(\bmod 2)$, see Figure 2 as an example, $C N C_{4}[n]=\bigcup_{i=1}^{n} T_{i}$, where $T_{i}$ is a partition of the molecular graph $\mathrm{CNC}_{4}[n]$. There are four classes of vertices for each section of $T_{i}$. We have 4 
vertices with $M(u)=6$, and $4 n$ vertices meeting $M(u)=9$ of class 1 have mean eccentric connectivity $3 n+2$, and $8(n-2 l-1)$ vertices for $0 \leq l \leq(n-2) / 2$ satisfy $M(u)=9$ of class 2. And the eccentric connectivity of $4(n-2 l-1)$ vertices of this class is equals to $3 n-2 l+1$ and the eccentric connectivity of other vertices of this class is equals to $3 n-2 l$. For $l \leq k \leq l+1$, if $k=l$ then there are $8 l+8$ vertices having $M(u)=9$ and eight vertices meeting $M(u)=6$. If $k-l=1$ then there are $8 l+8$ vertices satisfying $M(u)=9$ and 8 vertices with $M(u)=7$ and $e c(u)=4 n-l-k$ where $0 \leq l \leq(n-4) / 2$. Also there are eight vertices meeting $M(u)=5$ and $e c(u)=4 n+2$ and eight vertices satisfying $M(u)=7$ and $e c(u)=4 n+1$. Thus, using the definition of modified eccentric connectivity index and modified eccentric connectivity polynomial, we get

$$
\begin{gathered}
\zeta_{c}\left(C N C_{4}[n]\right)=\sum_{v \in V\left(C N C_{4}[n]\right)} M(v) e c(v)=\sum_{k=0}^{1} 8(2 k+5) \cdot(4 n+2-k)+ \\
\sum_{l=0}^{\frac{n-4}{2}} \sum_{k=l}^{l+1}((8 l+8) \cdot 9+8(6+k-l)) \cdot(4 n-k-l)+(36 n+24) \cdot(3 n+2)+ \\
\sum_{l=0}^{\frac{n-2}{2}} \sum_{k=0}^{1} 9(3 n-k-2 l+1) \cdot(4 n-8 l-4)=108 n^{3}+236 n^{2}+150 n+32 \\
\zeta_{c}\left(C N C_{4}[n], x\right)=\sum_{v \in V\left(C N C_{4}[n]\right)} M(v) x^{e c(v)}=\sum_{k=0}^{1} 8(2 k+5) x^{4 n+2-k}+ \\
\frac{n-4}{\sum_{l=0}^{2}} \sum_{k=l}^{l+1}(64 l+120+8 k) x^{4 n-k-l}+(36 n+24) x^{3 n+2}+\sum_{l=0}^{\frac{n-2}{2}} \sum_{k=0}^{1} 9(3 n-k-2 l+1) x^{4 n-8 l-4}
\end{gathered}
$$

\section{Conclusion}

In our article, by virtue of the molecular graph structural analysis and mathematical derivation, we mainly report the modified eccentric connectivity index and modified eccentric connectivity polynomial of tetragonal carbon nanocones $C N C_{4}[n]$. As the modified eccentric connectivity index and modified eccentric connectivity polynomial are widely used in the analysis of oxidation procedure for chemical compounds, the theoretical conclusion obtained in this article illustrates the promising prospects of the application to the chemical and pharmacy engineering.

Acknowledgments. First, we thank the reviewers for their constructive comments in improving the quality of this paper. This work was supported in part by the National Natural Science Foundation of China (61142007), the Open Research Fund by Jiangsu Key Laboratory of Recycling and Reuse Technology for Mechanical and Electronic Products (RRME-KF1612) and the Natural Science Foundation of Jiangsu University of Technology in China (KYY14013).

\section{References}

1. M. R. Farahani, W. Gao, "On Multiplicative and Redefined Version of Zagreb Indices of V-Phenylenic Nanotubes and Nanotorus", British Journal of Mathematics \& Computer Science, vol. 13, no. 5, pp. 1-8, 2016.

2. M. R. Farahani, W. Gao, M. R. Rajesh Kanna, "On the Omega Polynomial of A Family of Hydrocarbon Moleculs 'Polycyclic Aromatic Hydrocarbons PAHk'", Asian Academic Research Journal of Multidisciplinary, vol. 2, no. 7, pp. 263-268, 2015.

3. M. R. Farahani, W. Gao, M. R. Rajesh Kanna, "The Connective Eccentric Index for An Infinite Family of Dendrimers", Indian Journal of Fundamental and Applied Life Sciences, vol. 5, no. S4, pp. 766-771, 2015.

4. M. R. Farahani, M. R. Rajesh Kanna, W. Gao, "The Edge-Szeged Index of the Polycyclic Aromatic Hydrocarbons PAHk", Asian Academic Research Journal of Multidisciplinary, vol. 5, no. 6, pp. 136-142, 2015. 
5. M. R. Farahani, W. Gao, "The multiply version of Zagreb indices of a family of molecular graph", Journal of Chemical and Pharmaceutical Research, vol. 7, no. 10, pp. 535-539, 2015.

6. M. R. Farahani, W. Gao, "The Schultz Index and Schultz Polynomial of the Jahangir Graphs $\mathrm{J}_{5, m}$,", Applied Mathematics, no. 6, pp. 2319-2325, 2015.

7. M. R. Farahani, M. R. Rajesh Kanna, W. Gao, "The Schultz, modified Schultz indices and their polynomials of the Jahangir graphs Jn,m for integer numbers $n=3, m \geq 3$ ", Asian Journal of Applied Sciences, vol. 3, no. 6, pp. 823-827, 2014.

8. W. Gao, M. R. Farahani, "Degree-based indices computation for special chemical molecular structures using edge dividing method", Applied Mathematics and Nonlinear Sciences, vol. 1, no. 1, pp. 94-117, 2015.

9. W. Gao, M. R. Farahani, "The Theta polynomial $\Theta(G, x)$ and the Theta index $\Theta(G)$ of molecular graph Polycyclic Aromatic Hydrocarbons PAHk", Journal of Advances in Chemistry, vol. 12, no. 1, pp. 39343939, 2015.

10. W. Gao, Y. Gao, "A Note on Connectivity and $\lambda$-Modified Wiener Index", Iranian Journal of Mathematical Chemistry, vol. 6, no. 2, pp. 137-143, 2015.

11. J. A. Bondy, U. S. R. Mutry. "Graph Theory", Springer, Berlin, pp. 1-40, 2008.

12. A. R. Ashrafi, M. Ghorbani, "A study of fullerenes by MEC polynomials", Electronic Materials Letters, vol. 6, no. 2, pp. 87-90, 2010.

13. M. Alaeiyan, J. Asadpour, R. Mojarad, "A numerical method for MEC polynomial and MEC index of one-pentagonal carbon nanocones", Fullerenes, Nanotubes and Carbon Nanostructures, vol. 21, no. 10, pp. 825-835, 2013.

14. A. R. Ashrafi, M. Ghorbani, M. A. Hossein-Zadeh, "The eccentric connectivity polynomial of some graph operations", Serdica Journal of computing, vol. 5, no. 2, pp. 101-116, 2011.

15. N. Trinajstic, "Chemical Graph Theory". CRC Press, Boca Raton, 1992.

16. V. Kumar, A. K. Modan, "Apllication of graph theory: Models for prediction of carbonic anhydrase inhibitory activity of sulfonamides", J Math Chem., no. 42, pp. 925-940, 1991. 